\title{
Association between RNF213 c.14576G >A Variant (rs112735431) and Peripheral Pulmonary Artery Stenosis in Moyamoya Disease
}

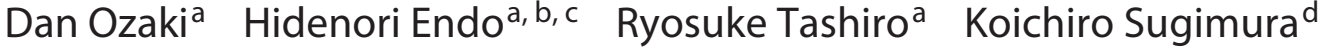 \\ Shunsuke Tatebe ${ }^{e}$ Satoshi Yasuda ${ }^{e}$ Yasutake Tomata $^{f}$ Toshiki Endo $^{a} g$

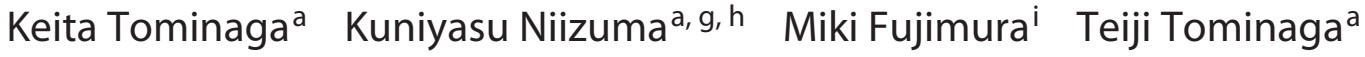 \\ aDepartment of Neurosurgery, Tohoku University Graduate School of Medicine, Sendai, Japan; bepartment of \\ Neurosurgery, Kohnan Hospital, Sendai, Japan; 'Division of Advanced Cerebrovascular Surgery, Tohoku University

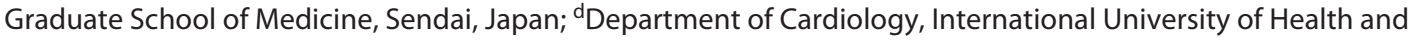 \\ Welfare Narita Hospital, Narita, Chiba, Japan; ${ }^{\text {DDepartment }}$ of Cardiovascular Medicine, Tohoku University Graduate \\ School of Medicine, Sendai, Japan; 'Faculty of Health and Social Services, Kanagawa University of Human Services, \\ Yokosuka, Japan; ${ }^{9}$ Department of Neurosurgical Engineering and Translational Neuroscience, Tohoku University \\ Graduate School of Medicine, Sendai, Japan; ' $\mathrm{D}$ epartment of Neurosurgical Engineering and Translational \\ Neuroscience, Graduate School of Biomedical Engineering, Tohoku University, Sendai, Japan; 'Department of \\ Neurosurgery, Hokkaido University Graduate School of Medicine, Sapporo, Japan
}

\section{Keywords}

RNF213 · Polymorphism · Homozygote · Moyamoya disease · Peripheral pulmonary artery stenosis

\begin{abstract}
Background: Moyamoya disease (MMD) and peripheral pulmonary artery stenosis (PPAS) are relatively rare and demonstrate steno-occlusive vascular lesions in different organs. Geneticstudiesidentified RNF213polymorphismc.14576G >A (rs112735431) as a susceptibility variant for East Asian MMD. RNF213 polymorphism c.14576G $>$ A is further associated with various vascular lesions of other organs. In this study, we aimed to clarify the incidence and clinical manifestations of PPAS in MMD patients and analyze the correlation between RNF213 genotype and PPAS. Methods: This retrospective case-control study investigated the association between RNF213 polymorphism and PPAS in $306 \mathrm{MMD}$ /quasi-MMD patients, reviewing the medical charts and imaging records of consecutive patients with MMD admitted from January
\end{abstract}

2015 to December 2020. Results: PPAS was observed in 3 $\mathrm{MMD}$ /quasi-MMD patients $(0.98 \%, 3 / 306)$. RNF213 polymorphism c.14576G >A was determined for all $306 \mathrm{MMD}$ /quasiMMD patients. The incidence of PPAS in RNF213-wildtype, RNF213-heterozygote, and RNF213-homozygote MMD/quasi-MMD patients was $0 \%(0 / 101), 0.5 \%(1 / 200)$, and $40 \%(2 / 5)$, respectively. The association between PPAS and homozygote polymorphism of RNF213 c.14576G >A was statistically significant in MMD/quasi-MMD patients ( $p=0.0018)$. In all cases, pulmonary artery hypertension due to PPAS was evident during their childhood and young adolescent stages. Surgical indications for MMD were discouraged in 1 case due to her severe cardiopulmonary dysfunction. Conclusions: The homozygote variant of RNF213 polymorphism c. $14576 \mathrm{G}>\mathrm{A}$ can be a potential predisposing factor for PPAS in MMD/quasi-MMD patients. Despite the relatively rare entity, PPAS should be noted to determine surgical indications for MMD/quasi-MMD patients.

(C) 2021 The Author(s) Published by S. Karger AG, Basel karger@karger.com www.karger.com/ced

Karger ${ }^{\prime \prime} \div$

BOPEN ACCESS
(C) 2021 The Author(s)

Published by S. Karger AG, Basel

This is an Open Access article licensed under the Creative Commons Attribution-NonCommercial-4.0 International License (CC BY-NC) (http://www.karger.com/Services/OpenAccessLicense), applicable to the online version of the article only. Usage and distribution for commercial purposes requires written permission.
Correspondence to:

Hidenori Endo, hideendo@gmail.com 


\section{Introduction}

Moyamoya disease (MMD) is a cerebrovascular disease characterized by chronic and progressive steno-occlusive lesions at the terminal portion of internal carotid arteries and the development of "Moyamoya vessels" [1, 2]. Although intracranial carotid arteries are preferentially affected in MMD, extracranial vascular involvement, such as stenotic lesions in pulmonary, coronary, and renal arteries, has been reported [3-6].

Genetic studies identified a single-nucleotide polymorphism c.14576G>A (rs112735431) in the RNF213 gene as a susceptibility variant for East Asian MMD [7, 8]. In particular, the homozygote variant of RNF213 c. $14576 \mathrm{G}>\mathrm{A}$ is associated with early and severe onset of MMD [9]. Recent studies revealed that RNF213 polymorphism c.14576G $>\mathrm{A}$ is associated with non-MMD stenoocclusive lesions in intracranial $[10,11]$ and coronary arteries [12]. In addition, MMD cases with pulmonary artery stenosis (PPAS) had homozygote polymorphism of RNF213 c.14576G>A [3-5]. This observation raised the hypothesis that $R N F 213$ polymorphism c.14576G $>$ A plays essential roles in the development of PPAS in MMD patients. In this study, we aimed to clarify the incidence and clinical manifestations of PPAS in MMD patients and analyze the correlation between RNF213 genotype and PPAS.

\section{Materials and Methods}

This is a retrospective case-control study. This study adhered to the Declaration of Helsinki and was approved by the Ethics Committee of Tohoku University (Approval No. 2016-1-212 and 2018-1-675) and Kohnan Hospital (Approval No. 2020-0520-03). Written informed consent was obtained from all the participants or their guardians.

\section{Diagnosis of MMD/Quasi-MMD and PPAS}

We retrospectively reviewed the medical charts and imaging records of consecutive patients with MMD who visited our institution from January 2015 to December 2020. Diagnosis of MMD and quasi-MMD was based on the diagnostic criteria of the Research Committee on Spontaneous Occlusion of the Circle of Wills of the Ministry of Health, Labor and Welfare of Japan [1,2]. The patients were diagnosed as quasi-MMD, if the patients showed angiographic findings equivalent to MMD but also had comorbidities, such as autoimmune diseases (for instance, rheumatoid arthritis, systemic lupus erythematosus, anti-phospholipid antibody syndrome, and Sjogren syndrome), neurofibromatosis type 1, meningitis, intracranial tumors, Down syndrome, and sickle cell anemia. A total of $533 \mathrm{MMD} /$ quasi-MMD (523 MMD and 10 quasi-MMD) patients were enrolled in this study. Examination of cardiopulmonary systems was conducted when patients manifested signs of cardiopulmonary dysfunction. PPAS was diagnosed based on the clinical findings, including cardiopulmonary symptoms and the findings of imaging studies such as CT pulmonary angiography, ventilation-perfusion scan, and conventional pulmonary angiography. Peripheral pulmonary artery hypertension (PAH) was defined by a mean pulmonary artery pressure $\geq 25 \mathrm{~mm} \mathrm{Hg}$ and a pulmonary arterial wedge pressure $\leq 15 \mathrm{~mm} \mathrm{Hg}$ at rest at right heart catheterization. Indication of balloon pulmonary angioplasty for PPAS was to decrease pulmonary arterial pressure, to relive of $\mathrm{PAH}$ symptoms, and to de-escalate $\mathrm{PAH}$-specific drugs.

\section{Identification of RNF213 Polymorphism c.14576G >A} (rs112735431)

We identified RNF213 gene polymorphism c.14576G $>\mathrm{A}$ (rs112735431) (https://www.ncbi.nlm.nih.gov/projects/SNP/snp_ ref.cgi?rs=112735431) for $306 \mathrm{MMD} /$ quasi-MMD (300 MMD and 6 quasi-MMD) patients who agreed with genetic analysis, as described previously $[13,14]$. In short, RNF213 polymorphism rs112735431 was identified using the TaqMan SNP genotyping assay (Assay ID: C_153120198_10; Applied Biosystems, Foster City, CA, USA). Extracted DNA from peripheral blood or saliva was used for analysis. The real-time polymerase reaction was performed on a StepOnePlus real-time polymerase chain reaction system (Applied Biosystems) as follows: primary denaturation at $95^{\circ} \mathrm{C}$ for $10 \mathrm{~min}$, followed by $40 \mathrm{cycles}$ of denaturation at $95^{\circ} \mathrm{C}$ for $15 \mathrm{~s}$ and subsequent annealing and extension at $60^{\circ} \mathrm{C}$ for $1 \mathrm{~min}$. The investigators who were blinded to patients' information determined genotypes using Applied Biosystems ${ }^{\circledR}$ TaqMan $^{\circledR}$ Genotype ${ }^{\mathrm{TM}}$ software.

\section{Statistical Analysis}

All statistical analyses were performed using IBM SPSS (version 24; IBM Corp). The association between RNF213 polymorphism c.14576G >A and the incidence of PPAS was evaluated by Fisher's exact test with Bonferroni correction. Significance was considered with 2 -sided $p$ values below 0.0167 .

\section{Results}

The Association between RNF213 Genotype and PPAS in $M M D /$ uasi-MMD Patients

A total of $306 \mathrm{MMD} /$ quasi-MMD patients (300 MMD and 6 quasi-MMD) agreed for genetic testing, which revealed heterozygote and homozygote polymorphism in 1 and $2 \mathrm{MMD}$ /quasi-MMD patients with PPAS, respectively (Table 1). The incidence of PPAS was higher in MMD/ quasi-MMD patients with homozygote polymorphism of RNF213 c.14576G >A $(40 \%, 2 / 5)$ than in those without polymorphism or with heterozygote polymorphism of RNF213 c. $14576 \mathrm{G}>\mathrm{A}(0 \%$ [0/101] and 0.5\% [1/200], respectively) (Table 1). Statistical analysis demonstrated the significant association between homozygote polymorphism of RNF213 c.14576G>A and PPAS in MMD/ quasi-MMD patients $(p=0.0018)$, whereas there is no significant association between PPAS and heterozygote polymorphism of $R N F 213$ c. $14576 \mathrm{G}>\mathrm{A}(p=1.000)$. 
Table 1. Frequency of RNF213 polymorphism c.14576G>A (rs112735431) in MMD/quasi-MMD patients associated with PPAS

\begin{tabular}{|c|c|c|c|}
\hline & \multirow{2}{*}{$\begin{array}{l}\text { RNF213-wildtype } \\
(n=101)\end{array}$} & \multicolumn{2}{|l|}{ RNF213-mutant } \\
\hline & & RNF213-heterozygote $(n=200)$ & RNF213-homozygote $(n=5)$ \\
\hline
\end{tabular}

MMD, Moyamoya disease; PPAS, peripheral pulmonary artery stenosis.

Table 2. Clinical characteristics of MMD/quasi-MMD patients associated with PPAS

\begin{tabular}{|c|c|c|c|c|c|c|c|c|}
\hline Case & Age & Sex & $\begin{array}{l}\text { MMD/ } \\
\text { quasi-MMD }\end{array}$ & $\begin{array}{l}\text { RNF213 } \\
\text { c. } 14576 \mathrm{G}>\mathrm{A}\end{array}$ & Family history & Clinical manifestation & Treatment (MMD) & Treatment (PPAS) \\
\hline 1 & 19 & $\mathrm{~F}$ & MMD & Homozygote & None & $\begin{array}{l}11 \mathrm{Y} \text { : cardiac murmur } \\
16 \mathrm{Y} \text { : TIA, shortness of breath }\end{array}$ & Antiplatelet therapy & $\begin{array}{l}\text { BPA, prostacyclin } \\
\text { agonist }\end{array}$ \\
\hline 2 & 56 & M & MMD & Homozygote & None & $\begin{array}{l}25 \text { Y: bloody sputum } \\
31 \text { Y: headache } \\
43 \text { Y: SAH }\end{array}$ & Routine follow-up & $\begin{array}{l}\text { BPA, prostacyclin } \\
\text { agonist }\end{array}$ \\
\hline
\end{tabular}

MMD, Moyamoya disease; PPAS, peripheral pulmonary artery stenosis; F, female; $M$, male; $Y$, year-old; TIA, transient ischemic attack; SAH, subarachnoid hemorrhage; BPA, balloon pulmonary angioplasty; HOT, home oxygen therapy.

\section{Clinical Manifestations in MMD/Quasi-MMD}

Patients with PPAS

Clinical characteristics of $3 \mathrm{MMD}$ /quasi-MMD patients with PPAS are summarized in Table 2. These 3 patients had a family history of MMD/quasi-MMD, of which 2 cases suffered from transient ischemic attacks (cases 1 and 3) and 1 patient had a history of subarachnoid hemorrhage (case 2). Only case 3 underwent revascularization for MMD/quasiMMD before the manifestation of cardiopulmonary dysfunction. All 3 cases suffered from peripheral PAH due to PPAS when they were children or young adolescents. A prostacyclin agonist was administered, and balloon pulmonary angioplasty was performed in all 3 cases. We conservatively treated MMD in cases 1 and 2, considering the surgical risk due to severe cardiopulmonary dysfunction. Neither ischemic nor hemorrhagic events recurred so far. A representative case (case 1) is shown in Figure 1.

\section{Discussion}

This study revealed a significant association between homozygote variant of RNF213 polymorphism c. $14576 \mathrm{G}>\mathrm{A}$ and PPAS in MMD/quasi-MMD patients. The homozygote variant of RNF213 polymorphism
c.14576G >A can be a potential predisposing factor for PPAS in MMD/quasi-MMD patients.

All the MMD cases with PPAS in the previous literature studies [3-5] and the present study harbored homozygote polymorphism of RNF213 c.14576G >A, except for 1 quasi-MMD case with heterozygote polymorphism in the present study. Likewise, a recent prospective study revealed the association among extracranial arteriopathies and RNF213 polymorphism, especially the homozygote variant [6]. However, it did not include MMD patients associated with PPAS probably due to small number of enrolled cases. These data support the hypothesis that RNF213 polymorphism c. $14576 \mathrm{G}>\mathrm{A}$, especially the homozygote variant, can be the predisposing factor for various steno-occlusive vascular lesions [15].

RNF213 mediates various aspects of cellular homeostasis. Experimental studies have revealed the physiological roles of RNF213 in nonmitochondrial oxygen consumption [16], lipid metabolism [17], and antigen uptake, processing, and presentation [18]. Given the observations that knock-in of the RNF213 polymorphism c. $14576 \mathrm{G}>\mathrm{A}$ variant itself could not lead to the development of MMD [19], environmental stimuli could potentiate the endothelial dysfunction and proangiogenic activity. Intrinsic chronic inflammatory state can be one of the important environ- 

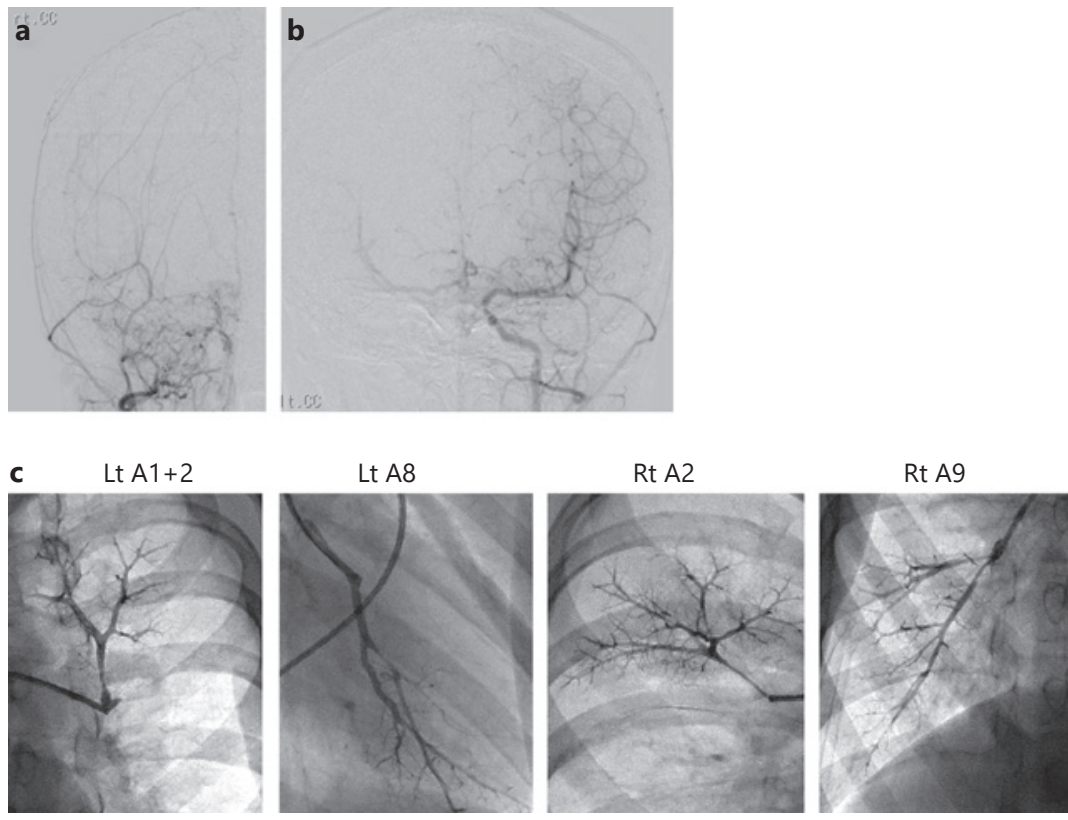

Fig. 1. Representative case: MMD which exhibited pulmonary hypertension due to peripheral pulmonary artery stenosis (case 1). A 16-year-old female patient with frequent transient ischemic attack in her right extremities and exertional dyspnea was diagnosed as MMD and PAH due to peripheral pulmonary artery stenosis. Genetic analysis identified the homozygote polymorphism of RNF213 c.14576G $>$ A. Decreased cerebral blood flow suggested the need of revascularization surgery, but severe cardiopulmonary dysfunction discouraged us to perform surgery for MMD. We continued antiplatelet therapy for MMD and started prostacyclin agonist. Then, we performed multiple balloon pulmonary angioplasty to

mental stimuli, as evidenced by high prevalence of co-existing autoimmune diseases $[13,20]$ and increased levels of circulating inflammatory factors [21-23]. Experimental studies showing the impaired $\mathrm{T}$-cell response and enhanced angiogenic activities in Rnf213-mutant mice also support this idea $[18,24,25]$. Despite the need of the further investigations, RNF213 appears to modulate metabolism and immune systems, which may affect vascular pathology.

Neurosurgical indications should be carefully determined if $\mathrm{PAH}$ is evident in MMD patients. Tokunaga et al. [3] reported 1 MMD case who passed away postoperatively due to the severe pneumonia associated with PPAS. This case suggests neurosurgical interventions are challenging once $\mathrm{PAH}$ is evident. In the present study, surgical treatment for an ischemic-onset MMD patient (case 1) was not performed due to severe cardiopulmonary improve PAH. The patient manifested neither further ischemic attacks nor the worsening of cardiopulmonary dysfunction. a Right common carotid artery angiography showing the occlusion of the right internal carotid artery and the development of Moyamoya vessels with transdural anastomosis. b Left common carotid artery angiography showing slight stenotic lesion at the terminal portion of the left internal carotid artery. c Pulmonary angiography showing multiple segmental stenotic lesions in peripheral pulmonary arteries. MMD, Moyamoya disease; PAH, pulmonary artery hypertension.

dysfunction. Fortunately, this case remained free from further ischemic attacks after antiplatelet therapy. A thorough follow-up of both MMD and PAH is warranted because the progression of PPAS can affect cerebral ischemia. These observations suggest the necessity of careful management in MMD/quasi-MMD patients with PPAS.

This retrospective study holds several limitations. First, the incidence of PPAS might be underestimated because cardiopulmonary system examination was performed only when the patients developed any symptoms, including cardiac murmur. Second, sampling bias may exist due to the availability of genetic testing and the lack of asymptomatic MMD/quasi-MMD patients in the present study. A future prospective study which screens PPAS in all the MMD/quasi-MMD cases is required to validate the findings of the current study. 


\section{Conclusion}

There was a significant association between homozygote variant of RNF213 polymorphism c.14576G $>$ A and PPAS in MMD/quasi-MMD patients. The homozygote variant of $R N F 213$ polymorphism c. $14576 \mathrm{G}>\mathrm{A}$ can be a potential predisposing factor for PPAS in MMD/quasiMMD patients. Despite the relatively rare entity, PPAS should be noted to determine surgical indications for $\mathrm{MMD} /$ quasi-MMD patients.

\section{Statement of Ethics}

This study adhered to the Declaration of Helsinki and was approved by the Ethics Committee of Tohoku University (Approval No. 2016-1-212 and 2018-1-675) and Kohnan Hospital (Approval No. 2020-0520-03). Written informed consent was obtained from all the participants or their guardians.

\section{Conflict of Interest Statement}

The authors have no conflicts to declare.

\section{Funding Sources}

This work was supported by the Ministry of Health, Labor and Welfare of Japan Grant No. S17310031 and the Japan Society for the Promotion of Science KAKENHI Grant No. 17K10815, 20K09362, and 21H04835.

\section{Author Contributions}

H.E., D.O., and R.T. contributed to conception and design. D.O., R.T., H.E., K.T., and M.F. contributed to genetic testing. H.E., K.S., S.T., T.E., K.N., and M.F. contributed to diagnosis and treatment of MMD patients with PPAS. D.O., H.E., R.T., and K.T. contributed to interpretation of the data. R.T. and Y.T. contributed to statistical analysis. D.O. and R.T. contributed to drafting. K.N., M.F., and T.T. contributed to funding acquisition. H.E., S.K., S.T., S.Y., Y.T., T.E., M.F., K.N., and T.T. contributed to critical revision of the manuscript. S.Y. and T.T. contributed to supervision. All authors read and approved the final version of the manuscript.

\section{Data Availability Statement}

All data generated or analyzed during this study are included in this article. Further enquiries can be directed to the corresponding author.

\section{References}

1 Research Committee on the Pathology and Treatment of Spontaneous Occlusion of the Circle of Willis; Health Labour Sciences Research Grant for Research on Measures for Intractable Diseases. Guidelines for diagnosis and treatment of moyamoya disease (spontaneous occlusion of the circle of willis). Neurol Med Chir. 2012;52:245-66.

2 Tominaga T, Suzuki N, Miyamoto S, Koizumi A, Kuroda S, Takahashi JC, et al. Recommendations for the management of moyamoya disease: a statement from Research Committee on Spontaneous Occlusion of the Circle of Willis (moyamoya disease) [2nd edition]. Surg Cereb Stroke. 2018;46:136-40. (Jpn).

3 Tokunaga K, Hishikawa T, Sugiu K, Date I. Fatal outcomes of pediatric patients with moyamoya disease associated with pulmonary artery hypertension. Report of two cases. Clin Neurol Neurosurg. 2013;115:335-8.

4 Fukushima H, Takenouchi T, Kosaki K. Homozygosity for moyamoya disease risk allele leads to moyamoya disease with extracranial systemic and pulmonary vasculopathy. Am J Med Genet A. 2016;170:2453-6.

5 Chang S, Song JS, Park TK, Yang YH, Kwon WC, Kim SR, et al. Nonsyndromic peripheral pulmonary artery stenosis is associated with homozygousity of RNF213 p.R4810Lys regardless of co-occurrence of moyamoya disease. Chest. 2018;153:404-13.

6 Lee TK, Yeon JY, Kim SM, Bang OY, Kim JS, Hong SC. Prospective screening of extracranial systemic arteriopathy in young adults with moyamoya disease. J Am Heart Assoc. 2020;9:e016670.

7 Kamada F, Aoki Y, Narisawa A, Abe Y, Komatsuzaki S, Kikuchi A, et al. A genome-wide association study identifies RNF213 as the first moyamoya disease gene. J Hum Genet. 2011;56:34-40.

8 Liu W, Morito D, Takashima S, Mineharu Y, Kobayashi $\mathrm{H}$, Hitomi $\mathrm{T}$, et al. Identification of RNF213 as a susceptibility gene for moyamoya disease and its possible role in vascular development. PLoS One. 2011;6:e22542.

9 Miyatake S, Miyake N, Touho H, Nishimura-Tadaki A, Okada I, Tsurusaki Y, et al. Homozygous c. $14576 \mathrm{G}>\mathrm{A}$ variant of RNF213 predicts early-onset and severe form of moyamoya disease. Neurology. 2012;78: 803-10.

10 Miyawaki S, Imai H, Shimizu M, Yagi S, Ono $\mathrm{H}$, Mukasa A, et al. Genetic variant RNF213 c. $14576 \mathrm{G}>\mathrm{A}$ in various phenotypes of intracranial major artery stenosis/occlusion. Stroke. 2013;44:2894-7.

11 Okazaki S, Morimoto T, Kamatan Y, Kamimura T, Kobayashi $\mathrm{H}$, Harada $\mathrm{K}$, et al. Moyamoya disease susceptibility RNF213 p.R4810K increases the risk of ischemic stroke attributable to large-artery atherosclerosis. Circulation. 2019;139:295-8.

12 Morimoto T, Mineharu Y, Ono K, Nakatochi M, Ichihara S, Kabata R, et al. Significant association of RNF213 p.R4810K, a moyamoya susceptibility variant, with coronary artery disease. PLoS One. 2017;12:e175649.
13 Tashiro R, Niizuma K, Khor SS, Tokunaga K, Fujimura M, Sakata $\mathrm{H}$, et al. Identification of HLA-DRB $1 * 04: 10$ allele as risk allele for Japanese moyamoya disease and its association with autoimmune thyroid disease: a case-control study. PLoS One. 2019;14(8):e0220858.

14 Tashiro R, Fujimura M, Katsuki M, Nishizawa T, Tomata Y, Niizuma K, et al. Prolonged/delayed cerebral hyperperfusion in adult patients with moyamoya disease with RNF213 gene polymorphism c.14576G $>$ A (rs112735431) after superficial temporal artery-middle cerebral artery anastomosis. J Neurosurg. 2020 Oct 23: 1-8. Epub ahead of print.

15 Bang OY, Chung JW, Kim DH, Won $\mathrm{HH}$, Yeon JY, Ki CS, et al. Moyamoya disease and spectrums of RNF213 vasculopathy. Transl Stroke Res. 2020;11:580-9.

16 Bahn R, Iorio C, Marcotte R, Xu Y, Cojocari D, Rahman AA, et al. PTP1B controls non-mitochondrial oxygen consumption by regulating RNF213 to promote tumour survival during hypoxia. Nat Cell Biol. 2016;18:803-13.

17 Sugihara M, Morito D, Ainuki S, Hirano Y, Ogino $\mathrm{K}$, Kitamura $\mathrm{A}$, et al. The AAA+ ATPase/ubiquitin ligase mysterin stabilizes cytoplasmic lipid droplets. J Cell Biol. 2019; 218:949-60.

18 Tashiro R, Niizuma K, Kasamatsu J, Okuyama Y, Sherif R, Kikuchi A, et al. Dysregulation of Rnf213 gene contributes to T cell response via antigen uptake, processing and presentation. J Cell Physiol. 2021 May 10. Epub ahead of print. 
19 Kanoke A, Fujimura M, Niizuma K, Ito A, Sakata H, Sato-Maeda M, et al. Temporal profile of the vascular anatomy evaluated by 9.4-tesla magnetic resonance angiography and histological analysis in mice with the R4859K mutation of RNF213, the susceptibility gene for moyamoya disease. Brain Res. 2015;1624:497-505.

20 Bower RS, Mallory GW, Nwojo M, Kudva YC, Flemming KD, Myer FB. Moyamoya disease in primarily white, midwestern US population: increased prevalence of autoimmune disease. Stroke. 2013;44:1997-9.
21 Fujimura M, Watanabe M, Narisawa A, Shimizu $\mathrm{H}$, tominaga $\mathrm{T}$. Increased expression of serum matrix metalloproteinase- 9 in patients with moyamoya disease. Surg Neurol. 2009; 72:476-80.

22 Kang HS, Kim JH, Phi JH, Kim YY, Kim JE Wang KC, et al. Plasma matrix metalloproteinases, cytokines and angiogenic factors in moyamoya disease. J Neurol Neurosurg Psychiatry. 2010;81:673-8.

23 Fujimura M, Fujimura T, Kakizaki A, SatoMaeda M, Niizuma K, Tomata Y, et al. Increased serum production of soluble CD163 and CXCL5 in patients with moyamoya disease: involvement of intrinsic immune reaction in its pathogenesis. Brain Res. 2018;1679: $39-44$.
24 Ito A, Fujimura M, Niizuma K, Kanoke A, Sakata H, Morita-Fujimura Y, et al. Enhanced post-ischemic angiogenesis in mice lacking RNF213; a susceptibility gene for moyamoya disease. Brain Res. 2015;1594:310-20.

25 Kanoke A, Fujimura M, Niizuma K, Fujimura T, Kakizaki A, Ito A, et al. Temporal profile of magnetic resonance angiography and decreased ratio of regulatory T cells after immunological adjuvant administration to mice lacking RNF213, a susceptibility gene for moyamoya disease. Brain Res. 2016;1642:1-9. 